\title{
EL DESARROLLO PSÍQUICO: PROBLEMAS Y SOLUCIONES
}

\author{
THE DEVELOPMENT PSYCHIC: PROBLEMS AND SOLUTIONS
}

Marta Shuare $^{1}$

\begin{abstract}
Resumen
Se analiza el problema del desarrollo psíquico y se señala la importancia que tiene su comprensión para enfocar el proceso educativo. Se resumen los dos enfoques tradicionales sobre los determinantes del desarrollo y se exponen los principales postulados de la teoría histórico-cultural de L. S. Vigotski sobre el desarrollo psíquico. Se describen las funciones psíquicas superiores, su origen y formación.
\end{abstract}

Palabras-claves: Teoría histórico-cultural. Funciones psíquicas superiores. Instrumentos. mediatización. signos.

\begin{abstract}
The article analyzes the problem of the psychic development and highlights the importance of its understanding to focus the educational process. The two traditional approaches to the determinants of development are summarized and the main postulates of the historical-cultural theory of L. S. Vygotsky on psychic development are presented. The higher psychic functions, their origin and formation, are described.
\end{abstract}

Key-words: Historical-cultural theory. Superior psychic functions. Instruments. Mediation. Signs.

Uno de los problemas más importantes tanto para la psicología como para la pedagogía (entendida como una disciplina que se ocupa de las cuestiones relativas a la enseñanza y el aprendizaje, sea de infantes, niños, adolescentes y/o adultos) es el del desarrollo psíquico.

De la concepción que se tenga sobre el desarrollo de los procesos psíquicos, en qué consiste, de qué factores depende, a qué influencias está sometido y cuáles son sus principales características en las diferentes etapas de la vida del sujeto, dependerá, entre otras cosas, el enfoque que se dé al proceso educativo.

\footnotetext{
${ }^{1}$ Ph.D. en psicología por la Universidad estatal de Moscú.
} 
Dicho con otras palabras, la concepción sobre el desarrollo psíquico es el fundamento de las ideas y teorías sobre la enseñanza y el aprendizaje, de los métodos de enseñanza que se elaboran para asegurar el proceso educativo, así como de los criterios sobre su mayor o menor éxito.

Aunque los profesionales ocupados en la educación no siempre lo acepten, está claro que la pedagogía, en todas sus manifestaciones y aplicaciones, se construye sobre la base de las concepciones a las que se adhiera, en forma implícita o explícita, sobre los procesos psíquicos, su estructura, modificaciones y cambios; en una palabra sobre la esencia de la psiquis.

Uno de los interrogantes que surgen de este planteo es el referido al desarrollo de las capacidades en los niños, tema fundamental para cualquier pedagogía y directamente dependiente de la concepción general sobre la psiquis y su desarrollo: según se entienda cómo y por qué factores están determinadas las capacidades del sujeto, tendremos respuestas pedagógicas diferentes en lo que concierne a en qué consiste el proceso educativo, cómo debe organizarse, así como cuáles son los métodos adecuados para obtener los resultados deseados.

Existen dos enfoques tradicionales sobre la cuestión de los determinantes del desarrollo: el bio-genético y el socio-genético, igualmente mecanicistas y, en última instancia, reduccionistas.

Si sostenemos cualquiera de estos enfoques sobre el desarrollo psíquico del sujeto, el problema será resuelto de manera unilateral: la oposición "bio"-"socio" implica la existencia de dos realidades autónomas y dicotómicas, que no se unen más que con la conjunción " $y$ ".

No se trata de "tomar un poquito de aquí y otro poquito de allá": esto conduce a que la desnudez de la determinación del desarrollo por el nivel biológico se cubra púdicamente con los vestidos de las condiciones socioculturales. Y la imagen no es inocente: los vestidos cubren, pero no se integran al cuerpo. A veces solo lo embellecen un tanto; otras, ocultan los defectos, pero no los modifican.

Este es el resultado al que se condena todo eclecticismo; a saber, la unión de diferentes ideas, puntos de vista, etc., como resultado de lo cual se alcanzan conclusiones superficiales, pero que aparentemente son confiables.

Puede que cualquiera de estas variantes sea considerada por algunos científicos suficiente para dilucidar el problema del desarrollo del individuo humano. Un claro ejemplo de ello son los intentos de explicar las conductas más complejas del hombre y de los animales por la presencia y la acción de genes, de programas genéticos y de estructuras micro-anatómicas, dotados de una extraordinaria "sabiduría", cuyo origen es misterioso (puede que sea dios, la naturaleza o cualquier 
otra "fuerza" desconocida). Si no hace mucho se trató de encontrar en del cerebro humano la neurona que responde a la palabra "mamá", los avances de las neurociencias permiten buscar ahora los genes responsables de la alegría, la melancolía, las depresiones, la felicidad conyugal o cualquier otro fenómeno tan "sencillo" como los citados.

Cabe reflexionar sobre las connotaciones ideológicas de semejante enfoque que, si bien no es de conocimiento general y común para la mayoría de la gente, "gracias" a los esfuerzos de los medios de comunicación y desinformación llegan, más o menos deformados a la conciencia del ciudadano: si todo depende de los genes que tenemos en nuestro organismo, solo cabe "seguir sus dictados" y someterse a su acción e influencia: no seríamos responsables de nuestra conducta, no estaríamos en condiciones de ofrecer resistencia a las circunstancias adversas con las que, con mucha frecuencia, el medio en el que vivimos nos enfrenta. En una palabra, no seríamos libres para determinar nuestra conducta, ni responsables de las acciones que emprendemos.

Sin embargo, existe una teoría que, por su fundamentación, sus postulados generales y particulares, su concepción sobre la esencia de los procesos psíquicos y su desarrollo, revierte esta situación y nos infunde optimismo sobre el destino del hombre y de la sociedad: se trata de la teoría histórico-cultural de L. S. Vigotski, la que es, a mi juicio, la concepción que da respuesta a los interrogantes sobre el desarrollo psíquico infantil y, consecuentemente, a los problemas de la enseñanza y del aprendizaje sin caer en la falsa oposición que mencionamos más arriba ni en posiciones reduccionistas $\mathrm{y}$, menos aun, pesimistas.

Era necesario encontrar un vector capaz de integrar (no sumar, no unir externamente) los componentes iniciales y que superara la dicotomía o la subordinación mecánica de los enfoques que hemos señalado.

Ese vector o eje central y centralizador es la historia. Ya bosquejado en la escuela francesa por Janet, en la teoría de Vigotski alcanza su plena significación, siendo abordado desde el marxismo (materialismo histórico). Sabemos que L. S. Vigotski conocía muy bien la obra de Marx que aplica creativamente para resolver los problemas del desarrollo de la psiquis humana.

Así, pues, se trata de la historia y no del tiempo: el desarrollo de los procesos psíquicos no consiste en el mero transcurrir; no despliegan sus características intrínsecas a medida que llega el momento para ello, como ocurre con la semilla que madura. En el caso de la semilla, el tiempo actúa "desde afuera": constituye solo el lapso necesario para que las estructuras internas se manifiesten, es el tiempo pasivo de la espera. 
Estamos en presencia del primer postulado esencial de la teoría vigotskiana: el tiempo humano, interpretado desde el punto de vista del materialismo, es historia, es decir el desenvolvimiento del proceso de la actividad productiva de las personas, de la sociedad.

No se trata de una simple enunciación, de una afirmación sin consecuencias. De ella se desprende que esa actividad productiva transforma no solo la naturaleza, sino también a los propios sujetos que la realizan.

¿Cómo ocurre que esa actividad productiva, transformadora de la naturaleza (a diferencia del animal, que la utiliza), modifica al mismo tiempo a quienes la llevan a cabo?

La actividad productiva humana se caracteriza por ser mediatizada: el instrumento se "interpone" entre el sujeto y el objeto de la actividad. Los instrumentos van conformando una segunda naturaleza y esta es de carácter social. Los objetos, creados por el trabajo, que se interponen entre el hombre y la naturaleza constituyen la cultura. Lo que se denomina "social" en el hombre no es un agregado, una "capa" superpuesta a la naturaleza "natural", como si fuera una torta (un bolo), sino que la transforma sometiéndola a leyes de orden superior. Dice L. S. Vigotski: "Por cuanto el desarrollo orgánico (del niño) se realiza en un medio cultural, se convierte en un proceso biológico históricamente condicionado". 1)

Subrayemos: no socialmente, sino históricamente condicionado. Y subrayemos también lo siguiente: "[...] el desarrollo orgánico [...] se realiza en un medio cultural [...]"

Esta aclaración es de extrema importancia: en primer lugar, permite afirmar que la denominación "socio-cultural" con la que, a veces, se identifica la teoría de Vigotski es incorrecta y conduce a una interpretación equivocada de la teoría en cuestión; en segundo lugar, la afirmación de Vigotski que hemos citado es esencial porque evita aquí la "trampa" de la doble determinación ("un poquito de esto y un poquito de aquello"), así como caer en la determinación unívoca por parte de lo social (en lo que incurrió la escuela sociológica francesa), que no deja lugar para la libertad creadora del hombre. En tercer lugar, Vigotski introduce y le otorga una importancia esencial, determinante, a los objetos de la cultura (los medios de producción, las herramientas y los instrumentos, los signos, etc.).

El segundo postulado esencial o generación conceptual que, en la teoría vigotskiana, produce el eje histórico es que los procesos psíquicos, la psiquis humana no son algo dado de una vez y para siempre, sino que existe un desarrollo histórico de los mismos, una dependencia con respecto a la vida y actividad de los hombres. La historia de la psiquis humana es la historia social 
de su constitución. Una vez constituida, no está dada de manera directa en las estructuras orgánicas del niño, sino que está presupuesta: el sujeto deberá apropiarse (adquirirla, asimilarla) en el curso de su vida y actividad.

Así como la psiquis no es algo inmutable e invariable en el curso del desarrollo histórico de la sociedad, no lo es tampoco en el curso el desarrollo individual.

El tercer postulado que se deriva de la historicidad es el del carácter mediatizado de la psiquis humana. A diferencia de los fenómenos psíquicos naturales de los animales, los fenómenos psíquicos humanos (las "funciones psíquicas superiores") son producto de la compleja interacción el sujeto con el mundo, interacción mediatizada por los objetos creados por el hombre en el curso de la historia de la sociedad y, en particular, por los sistemas de signos. Así como los instrumentos de trabajo mediatizan las relaciones del hombre con la naturaleza, ciertos instrumentos especiales (genéricamente, los signos) se interponen entre la función psíquica natural y su objeto. Esta interposición cambia de raíz las propiedades de todos los procesos psíquicos.

Por último señalemos que la superación de la idea del carácter inmediato, dado, de la psiquis, permite superar los siguientes postulados de la psicología tradicional: 1) la psiquis es algo dado y no generado; 2) es algo dado al sujeto de alguna manera (por la acción de "leyes naturales"; por la acción de la voluntad divina; por cierto "fiat" de naturaleza desconocida, etc.); 3) se encuentra "dentro" del sujeto individual y cabe suponer o dudar de su existencia en otros seres semejantes; 4) es algo dado internamente, sea en las estructura cerebrales o en espacios virtuales dentro del individuo.

Vigotski plantea de forma absolutamente diferente el problema del "lugar" donde residen los procesos psíquicos y su modo de existencia: la psiquis existe en el espacio-tiempo de las interrelaciones del hombre con el mundo, con sus semejantes, en el espacio-tiempo de su actividad. Esta afirmación no es fácilmente comprensible, pues estamos acostumbrados a considerar el tiempo y el espacio como dos entidades separadas e independientes en las cuales transcurre la vida del hombre (nace y muere, sufre el transcurrir del tiempo, se desplaza en el espacio, etc.) y a pensar que la psiquis, los procesos psíquicos están "ubicados" en el interior del sujeto desde el inicio mismo y su génesis debiera buscarse en las "profundidades" del individuo (sea la glándula pineal, sean las estructuras celulares del cerebro, sea la existencia innata de estructuras dadas por la naturaleza). 
"Historia del desarrollo de las funciones psíquicas superiores", que Vigotski terminó de escribir en el año 1931, es un trabajo de aquellos que ahora se denominan con el término "fundacional". No sólo resulta fundamental para comprender el enfoque histórico-cultural: es de tal riqueza conceptual que resultó una referencia obligada para quienes continuaron las investigaciones en el contexto de dicha teoría (por supuesto, con las innovaciones, disparidades, contradicciones, oposiciones, etc. que normalmente se producen cuando se trata de una verdadera escuela de pensamiento e investigación). Me refiero a la teoría de la actividad de A. N. Leóntiev; a la concepción de la formación por etapas de las acciones mentales de P. Ya. Galperin; a los estudios sobre la psiquis infantil de D. B. Elkonin; a la creación y desenvolvimiento de la neuropsicología de A. R. Luria, a las investigaciones de L. I. Bozhóvich sobre el desarrollo de la personalidad, para mencionar sólo a sus discípulos y colaborados más conocidos.

Vigotski emprendió el análisis multifacético del proceso de formación de las funciones psíquicas superiores, tanto desde el punto de vista de su génesis como de su estructura general y particular para cada función, así como también del problema del método adecuado para investigarlas.

La primera idea central de este trabajo es la siguiente: hasta el momento, la psicología tradicional ha estudiado las funciones psíquicas superiores como procesos y formaciones naturales, siendo incapaz de tratarlas como productos del desarrollo histórico.

Al considerarlas "productos" naturales, la psicología tradicional no pudo aclarar ni explicar las diferencias entre los procesos propios del desarrollo orgánico y los propios del desarrollo cultural. En consecuencia, la psicología clásica no ha podido elucidar la diferencia entre "las dos series, diferentes en esencia, de regularidades" a las que están sometidos los procesos orgánicos y culturales de desarrollo. Así se produce la reducción de los procesos psíquicos complejos a los elementales y el estudio de las funciones psíquicas sólo en su aspecto natural.

El método aplicado por la psicología tradicional consiste en "desmenuzar" las funciones psíquicas superiores y las formas más complejas de comportamiento en sus elementos constituyentes: los procesos psíquicos superiores pierden las propiedades y regularidades que les son propias y son estudiados como si fueran la suma aritmética de sus elementos constitutivos.

Esta crítica a la base filosófica de la que se parte cuando se consideran los procesos psíquicos superiores como procesos naturales y cuando se los estudia mediante métodos de 
"simplificación", es aplicable tanto para la psicología subjetiva como para la psicología objetiva (el behaviorismo norteamericano y la reflexología rusa).

La segunda idea central de la "Historia del desarrollo de las funciones psíquicas superiores" es la necesidad de superar el reduccionismo, implícito cuando se las considera como funciones naturales. Vigotski señala que el reduccionismo es la consecuencia de enfocar este problema desde el punto de vista evolucionista, entendiendo por tal el tratamiento del desarrollo como un proceso lineal y paulatino.

La tercera idea central es erradicar definitivamente de la psicología la falaz idea del desarrollo como proceso lineal y paulatino.

Con esto no terminan las "desgracias" que trae a la psicología el tratar a las funciones psíquicas superiores como funciones naturales: la reducción de las estructuras complejas a sus elementos componentes, de las formas superiores de la psiquis a las inferiores, lleva consigo el estigma del dualismo: por cuanto por más reduccionismo que se aplique, las estructuras superiores de comportamiento, las formas complejas de la actividad psíquica, etc. siguen existiendo, habrá que introducir, en forma solapada o descubierta, una duplicidad esencial: la materia y el espíritu, los que, una vez desgajados, harán su camino paralelamente y hasta el infinito. Todas las reflexiones ontológicas y metodológicas de L. S. Vigotski están dedicadas a superar ese dualismo, que se caracteriza por: 1) el estudio de las funciones psíquicas superiores como si fueran procesos naturales; 2) la reducción de los procesos superiores y complejos a los elementales; 3) el desconocimiento de las regularidades y particularidades específicas del desarrollo cultural del comportamiento.

Estas características son propias tanto de la vieja psicología, subjetiva y empírica, como de la psicología objetiva, saber el conductismo norteamericano y la reflexología rusa. Pese a la radical y profundísima diferencia entre ellas, no puede perderse de vista ni por un momento que ambas corrientes están emparentadas por el mismo criterio metodológico, consistente en:

- aplicar, para el estudio de todo objeto, el enfoque analítico;

- suponer que la única forma de resolver los problemas a investigar es descomponer el objeto, el proceso o el fenómeno en elementos primarios;

- reducir las formas y las formaciones superiores a las inferiores;

- desconocer el aspecto cualitativo, que no puede ser reducido a diferencias cuantitativas. 
En una palabra, concluye Vigotski, estamos en presencia del carácter no dialéctico del pensamiento científico propio de dichas corrientes psicológicas.

Esto lleva a que el desarrollo de las funciones psíquicas superiores sea un área completamente ignorada como tal, que no existan métodos para investigar los problemas de este desarrollo, que no haya siquiera una delimitación clara de aspectos a estudiar. Más aun, no hay definiciones estrictas sobre qué son, en qué consisten, tratándoselas como si fueran las mismas funciones psíquicas inferiores o naturales, sólo que un poco "más complicadas".

Vigotski considera que es absolutamente errónea, por no reflejar la esencia del fenómeno estudiado, la idea central de todo enfoque asociacionista, reflexológico, etc., según la cual el desarrollo complejo se puede entender desmembrándolo en sus partes o elementos, de la misma forma que el resultado de una suma es la adición aritmética de los sumandos.

Si se examinan los problemas de las funciones psíquicas superiores como si fueran la prolongación de las mismas funciones naturales o inferiores, siempre podremos constatar que se reducen aquéllas a éstas, por más que se les agreguen adjetivaciones (como, por ejemplo: son las mismas funciones, pero "más complejas").

¿Por qué se produce esta reducción? La razón radica en que los investigadores no analizan estos procesos como hechos del desarrollo histórico del hombre y de la sociedad, en que no distinguen entre lo natural y lo cultural, entre lo biológico y lo social en el desarrollo psíquico. Los hechos del desarrollo orgánico y del desarrollo cultural se ubican en una misma serie y se los examina como si fueran fenómenos del mismo orden, de una misma naturaleza, con iguales regularidades.

Limitar la investigación del desarrollo psíquico el niño a sus primeros años de vida es la lógica consecuencia de considerar que ese desarrollo consiste en la maduración y ejercitación de las funciones naturales, elementales, dadas al organismo como ser biológico. Y que esa maduración y ejercitación llevarán (no se sabe cómo) a que el niño adquiera posteriormente todas las capacidades que le permitirán dominar el lenguaje, la lectura y la escritura, el pensamiento abstracto, las nociones matemáticas.

En realidad, sostiene Vigotski, la psicología infantil ha estudiado el estado embrionario de las funciones psíquicas superiores, "la embriología del espíritu humano".

Vigotski no rechaza ni desprecia el estudio de las formas primarias de la vida psíquica, sino que insiste en que es incorrecto superponer las funciones superiores a ellas. Se trata de estudiar 
cómo se produce la transformación esencial de las funciones psíquicas naturales, qué fuerzas impulsan esa transformación y cuáles son los medios que sirven a la misma.

En este sentido dice que, en la primera infancia, se hallan las raíces genéticas de las dos formas culturales fundamentales de la conducta: el empleo de herramientas y el lenguaje humano. Este hecho coloca a la primera infancia en el centro de la prehistoria del desarrollo cultural.

Tomemos nota de estas afirmaciones: "las dos formas culturales fundamentales [...]" y "la prehistoria del desarrollo cultural". Tenemos aquí dos momentos esenciales del análisis que hace Vigotski de la psicología tradicional en la cuestión del desarrollo psíquico y de su propia teoría. Señala que el vínculo entre el desarrollo natural y la conducta del niño, basados en la maduración de su aparato orgánico, y el desarrollo de las formas superiores de comportamiento no es de carácter evolutivo, paulatino, progresivo, sino revolucionario: se producen cambios bruscos y fundamentales del propio desarrollo, de las fuerzas que impulsan este proceso.

La psicología tradicional concibe el desarrollo de la conducta por analogía con el desarrollo embrionario, (es decir, orgánico, natural), ignorando así las características y propiedades del desarrollo psíquico.

Si la psicología empírica reduce el desarrollo psíquico del niño a la maduración de las funciones elementales $y$, por cuanto es indudable la existencia de funciones superiores, las agrega a las anteriores, sin indagar de dónde salen, la llamada psicología objetiva (reflexología, conductismo) se niega por principio a distinguir las funciones superiores de las inferiores y todo lo considera reacciones, sean ellas innatas o adquiridas (hábitos).

Se ignora o se reduce a fenómenos elementales aquello que constituye el hecho fundamental del desarrollo psíquico, a saber la formación de las funciones psíquicas superiores, así como sus características, las fuerzas motrices que impulsan su aparición y desenvolvimiento, las condiciones de su origen y surgimiento.

Siéndole ajeno a la psicología infantil tradicional el concepto mismo de desarrollo de las funciones psíquicas superiores, sostiene Vigotski, dicha psicología se ve forzada a limitar ese concepto al mero desarrollo biológico de las funciones elementales, que transcurre en directa dependencia de la maduración del cerebro, producto de la maduración orgánica del niño.

El hecho de que la psicología infantil sea incapaz de estudiar debidamente las funciones psíquicas superiores y se ocupe sólo del nivel inferior crea una brecha entre ella y la psicología 
general que, justamente, estudia las funciones psíquicas superiores (la atención voluntaria, la memoria lógica, etc.).

Ignorar el origen y el desenvolvimiento de éstas conduce a que se suponga que coexisten con las inferiores, como si fueran dos series paralelas e independientes una de la otra, sin vinculación genética, funcional ni estructural, como una dualidad dada originariamente, para la cual no hay explicación alguna.

El empirismo siempre da lugar al dualismo, subraya Vigotski en "Significado histórico de la crisis de la psicología". A su vez, esta dualidad entre lo superior y lo inferior, originada en el empirismo de la ciencia, lleva a la existencia de dos psicologías: la fisiológica, ciencia natural, explicativa, que construye sus proposiciones sobre la base de la relación causa-efecto; y la psicología comprensiva, descriptiva y teleológica.

La primera no puede ir más allá del estudio de procesos elementales y no puede servir de base para las ciencias humanas (la historia, la estética, las ciencias de la cultura, etc.), mientras que la segunda, ocupada en estas altas materias, no va más allá de la descripción de los fenómenos, por cuanto la explicación causal, única forma de existencia de las ciencias naturales, no es aplicable a estos fenómenos. El determinismo de las ciencias naturales hace imposible explicarlos, a menos que se los reduzca a sus elementos y pierdan así su esencia.

La segunda (la psicología del espíritu, comprensiva, teleológica) sostiene que 1) la psicología, por su misma esencia, no puede ser una disciplina de las ciencias naturales; 2) la vida del espíritu debe ser comprendida y no explicada; 3) el camino experimental e inductivo de la investigación debe ceder el lugar a la aprehensión intuitiva y a la comprensión de las esencias, al análisis de los datos inmediatos de la conciencia; 4) la explicación causal debe ser sustituida por la teleológica; 5) el materialismo de la psicología explicativa debe ser definitivamente eliminado; 6) en el estudio del espíritu es necesario renunciar a todos los vínculos materiales y a todos los métodos deterministas del pensamiento propios de la ciencia natural.

Así, pues, la psicología se encuentra en la disyuntiva (falsa y derivada del incorrecto planteo de la verdadera esencia de lo psíquico) de renunciar al estudio de los procesos psíquicos superiores o de renunciar a su explicación determinista-causal. Esta falsa disyuntiva se deriva de presuponer la existencia de regularidades de carácter puramente innato, natural, o puramente espiritual y metafísico, pero no regularidades históricas: se aceptan las leyes eternas de la naturaleza o las leyes eternas del espíritu, pero no se reconocen ni se admiten las leyes históricas. 
Vigotski emprende el intento de superar esta falsa alternativa, aplicando a la investigación científica el método dialéctico.

Para iniciar la exposición de sus ideas sobre la esencia del desarrollo psíquico, Vigotski reúne en un todo armónico tres conceptos fundamentales: el de función psíquica superior; el de desarrollo cultural de la conducta y el de adquisición (apropiación) de los propios procesos de comportamiento.

El concepto de "desarrollo de las funciones psíquicas superiores" comprende dos grupos de fenómenos que, a primera vista, parecen completamente heterogéneos, pero que en realidad representan dos ramas fundamentales, dos vertientes de desarrollo de las formas superiores de la conducta indisolublemente unidas, pero que nunca se funden. Se trata, en primer lugar, de los procesos de adquisición de los medios externos del desarrollo cultural y del pensamiento -el lenguaje, la escritura, el cálculo, el dibujo; en segundo lugar, de los procesos de desarrollo de las funciones psíquicas superiores específicas, llamadas atención voluntaria, memoria lógica, formación de conceptos, etc.

Se debe prestar especial atención al contenido de esta afirmación de Vigotski: los medios externos del desarrollo cultural y el desarrollo de las funciones psíquicas superiores forman lo que denomina proceso de desarrollo de las formas superiores de comportamiento.

Esto debe entenderse claramente en el sentido de que no hay desarrollo de las funciones psíquicas superiores sin la adquisición y dominio paulatino de los medios externos del desarrollo cultural. O sea que las FPS no "maduran" por sí mismas, porque el niño ha alcanzado una etapa dada de crecimiento, sino porque éste en el curso de su vida social (familia, escuela, grupos de pares, etc.) se va apoderando (apropiando) de dichos medios.

Con ello, se elimina cualquier interpretación 1) "sociologizante" de la teoría vigotskiana (la sociedad impondría en forma directa estas formas superiores de comportamiento) y 2) "espiritualizante" de dicha teoría, según la cual los medios externos, en particular el lenguaje, actuarían como demiurgos del desarrollo (negrita mía).

Es por eso que siempre consideré erróneo y deformante llamar a la teoría vigotskiana "sociohistórica". Por otra parte, destaquemos que no es fácil asimilar esta manera de plantear la cuestión: aquí es necesario ver y entender la relación dialéctica entre ambos términos, sin permitir al pensamiento "cotidiano" reducir uno al otro o dar una supremacía inexistente a alguno de ellos. 
Es necesario tener en cuenta, por un lado, que la evolución biológica de las especies animales llevó a la aparición del Homo Sapiens; por otra parte, esa especie, en el proceso del desarrollo histórico, se convirtió en un ser cultural. (F. Engels, en "El papel del trabajo en la transformación del mono en hombre", dice que el papel decisivo en esa evolución lo juegan las herramientas, los instrumentos de trabajo que permiten ya no el uso de la naturaleza, sino su transformación). Ambos procesos -el desarrollo biológico y el desarrollo cultural del comportamiento- están presentados en la filogénesis por separado, mientras que en la evolución del niño conforman un proceso único.

Importa destacar que el desarrollo de las funciones psíquicas superiores tiene lugar sin cambios en el tipo biológico humano; es decir queda fuera de los dictados de la evolución biológica.

En otras palabras, desde el Homo Sapiens a la actualidad lo que ha cambiado y cambia no es la organización biológica y fisiológica del hombre, producto del proceso evolutivo filogenético, sino el desarrollo de sus órganos artificiales, las herramientas, y, como consecuencia de ello, la organización de la conducta, en el curso del desarrollo histórico.

Una vez que el proceso evolutivo de la especie ha llegado al estadio del Homo Sapiens, ya no se modifica la organización biológica ni, en principio, el funcionamiento de los órganos constituidos en el curso de esa evolución biológica, es decir las funciones psicofisiológicas elementales. Lo que cambia sustancialmente en el proceso de desarrollo histórico son las funciones psíquicas superiores (el pensamiento verbal, la memoria lógica, la atención voluntaria, etc.)

Reiteremos esta afirmación, confirmada por todas las investigaciones arqueológicas y antropológicas: no hay diferencias fundamentales en el tipo biológico del hombre primitivo que puedan condicionar la diferencia entre su comportamiento y el comportamiento de representantes de épocas posteriores, entre el hombre actual y miembros de tribus aun existentes, como tampoco hay diferencias en sus funciones psíquicas elementales.

La evolución biológica de las especies y el proceso de desarrolllo histórico son dos líneas de desarrollo que, en el caso del niño, están fundidas en un proceso único.

Las diferencias que existen entre el comportamiento del hombre primitivo y el actual no pueden adjudicarse a causas biológicas. Las funciones psíquicas elementales o inferiores no son diferentes en uno y otro. Este hecho es fundamental y exige una explicación diferente a la que pudo dar hasta el momento la psicología sea de orientación biológica o la psicología asociacionista, que 
parte de la suposición de que las leyes psicológicas fundamentales son inmutables a lo largo del desarrollo histórico.

A lo largo de este proceso no se modifican sustancialmente las funciones psíquicas elementales, mientras que las superiores (memoria y atención voluntarias, pensamiento verbal, voluntad, etc.) cambian profundamente.

Si estos cambios no se deben a modificaciones de carácter biológico ¿qué las determina?

El desarrollo cultural del comportamiento contiene en sí el concepto de desarrollo de las funciones psíquicas superiores y la adquisición y empleo de los medios externos de comportamiento cultural (lenguaje, cálculo, escritura, dibujo, etc.).

La conclusión más general, dice Vigotski, es la siguiente: la cultura crea formas especiales de conducta, transforma la actividad de las funciones psíquicas, forma nuevos estadios en el comportamiento humano. En el proceso de desarrollo histórico el hombre cambia los modos y los métodos de su conducta, transforma las disposiciones y las funciones innatas, elabora y crea nuevas formas de comportamiento específicamente culturales.

EL uso de herramientas en el proceso de trabajo, que convierte la adaptación en transformación de la naturaleza, crea nuevas funciones y nuevas conductas.

La ontogénesis no repite la filogénesis ni la reproduce en ninguna medida; pero en ambas existen las dos líneas de desarrollo: la del comportamiento biológico (natural) y la del histórico (cultural), aunque la estructura y el contenido de ambos procesos son diferentes en la filo y la ontogénesis. Si en el primer caso ambas líneas existen por separado y están unidas por una relación de sucesión y continuidad, en la ontogénesis aparecen fundidas y formando un proceso único.

Según Vigotski, el desarrollo orgánico se produce en un medio cultural y, por eso mismo, se convierte en un proceso biológico históricamente condicionado. Llamo la atención sobre las palabras históricamente condicionado: Vigotski no dice socialmente condicionado, con lo que se distancia sustancialmente de la determinación mecánica de lo psíquico por los condicionamientos sociales; lo histórico es abarcativo de lo social; es, para decirlo figuradamente, un concepto "mayor" que lo social.

El hombre también está limitado en sus posibilidades de comportamiento: por ejemplo no puede volar; pero supera a todos los animales porque las amplía ilimitadamente mediante la creación y utilización de herramientas. 
Por ello, un momento decisivo en el desarrollo del niño, en el sentido de determinar las formas de comportamiento a su alcance, es cuando da el primer paso hacia el descubrimiento y uso independiente de herramientas, cosa que ocurre hacia fines del primer año de vida.

Cabe destacar que el sistema de la actividad del niño está doblemente determinado 1) por el grado de desarrollo orgánico y 2) por el grado de dominio de las herramientas o instrumentos culturales, creados a lo largo del desarrollo histórico de la humanidad. Estos dos sistemas se desarrollan conjuntamente, formando un tercer sistema, hecho que ocurre solo en la ontogénesis.

Vigotski reitera la afirmación de que lo biológico y lo cultural, tanto en el niño normal como anormal, son líneas de desarrollo heterogéneas, especiales, específicas; ellas no coexisten ni se superponen; no están relacionadas entre sí en forma mecánica y externa, sino que se entrelazan en una síntesis superior única.

Los estímulos artificiales creados por el hombre en el desarrollo histórico de la sociedad modifican sustancialmente su actividad material y espiritual y constituyen el rasgo distintivo de los procesos psíquicos humanos, de las funciones psíquicas superiores.

Dentro de esos estímulos artificiales se destacan los sistemas de signos (en particular, el lenguaje), el arte en todas sus manifestaciones, los símbolos, etc., lo que Vigotski denomina instrumentos, usando el término por analogía con los instrumentos materiales que mediatizan las relaciones entre el hombre y la naturaleza.

Vigotski concluye que "los estímulos artificiales creados por el hombre, que no guardan ninguna relación con la situación presente y han sido puestos al servicio de la adaptación activa, son el rasgo distintivo de las formas superiores de la conducta.

La creación y el uso de estímulos artificiales como medios auxiliares para el dominio de las propias reacciones sirven de base a la nueva forma de determinación de la conducta que distingue la conducta superior de la elemental: la presencia y el empleo de estímulos creados junto a los dados es, dice Vigotski, "el rasgo distintivo de la psicología humana".

En síntesis y sin explicitar en forma exhaustiva, en este artículo, todo el contenido de esta obra fundamental de L. S. Vigotski, podemos afirmar que la teoría histórico-cultural es una concepción que, explicitando dialécticamente los momentos de formación y desenvolvimiento de la psiquis humana, así como los factores que intervienen en ese desarrollo, pone a disposición de psicólogos y pedagogos una psicología que considera a la educación y la enseñanza factores 
decisivos en el desarrollo del ser humano y que, por su esencia misma, inspira esperanzas y optimismo en el destino del hombre y de la sociedad.

\section{REFERÊNCIAS}

VIGOTSKI, L. S. Historia del desarrollo de las funciones psíquicas superiores. Obras en 6 tomos. Moscú. Editorial Pedagógica. Tomo 3, 1983, p.6-386 (en ruso).

El significado histórico de la crisis de la psicología. Obras en 6 tomos. Moscú. Editorial Pedagógica. Tomo 1, p 291-437. (en ruso).

ENGELS, F. De las Obras escogidas (en tres tomos) de C. Marx y F. Engels. Editorial Progreso. Moscú, 1981. Tomo 3, p. 66-79 (en español). 